\title{
Prognostic factors in patients with septic shock in digestive surgery who have undergone direct hemoperfusion with polymyxin B-immobilized fibers: a retrospective observational study
}

\author{
Satoshi Matsukuma ${ }^{* *}$, Kazuhiko Sakamoto', Mitsuo Nishiyama', Takao Tamesa', Shigefumi Yoshino', \\ Shoichi Hazama', Rumi Oshibuchi², Norimasa Matsuda², Satoshi Matsumoto², Hiroya Wakamatsu², \\ Ryosuke Tsuruta ${ }^{3}$, Mishiya Matsumoto ${ }^{2}$ and Masaaki Oka ${ }^{1}$
}

\begin{abstract}
Background: Direct hemoperfusion with polymyxin B-immobilized fiber (PMX-DHP) has been widely used for patients with septic shock around the world, but the prognostic factors have not been fully understood. We conducted a retrospective analysis to determine the prognostic factors in patients with septic shock who underwent PMX-DHP.

Methods: Twenty-nine patients with septic shock who underwent PMX-DHP were included in the study. The patients were divided into groups based on survival $(n=23)$ and non-survival $(n=6) 28$ days after PMX-DHP, and the clinical data for the two groups before and after PMX-DHP were compared.

Results: In non-survivors, the vasopressor dependency index before PMX-DHP was significantly higher $(p=0.046)$, and the leukocyte count before PMX-DHP was significantly lower $(p=0.024)$ than in survivors. Furthermore, base excess after PMX-DHP was significantly lower in non-survivors $(p=0.007)$ than in survivors. The optimal cutoff points of the vasopressor dependency index, leukocyte count, and base excess identified by receiver operating characteristic curves were $0.499 / \mathrm{mmHg}, 1360 / \mathrm{LL}$, and $-6.4 \mathrm{mmol} / \mathrm{L}$, respectively. And the score using these three cutoffs, termed the prognostic score, was related to the prognosis of septic shock patients who underwent PMX-DHP (area under the curve $=0.946$ ).
\end{abstract}

Conclusions: The prognostic score, using three parameters which are immediately and readily available in early phase after starting PMX-DHP, might be useful to predict the prognosis of these patients.

Keywords: Intra-abdominal infection, Hemodynamic dysfunction, Prognostic score

\section{Background}

Sepsis is a systemic inflammatory response syndrome induced by infection [1]. Severe sepsis and septic shock affect 15 to 19 million people worldwide each year [2], mortality rate is declining as a result of advances in treatment, but is still $20 \%$ to $30 \%[3,4]$. Intra-abdominal infection is a main focus of severe sepsis and septic shock [5], and a crucial problem in surgical critical care. The Surviving Sepsis

\footnotetext{
* Correspondence: smatsu@yamaguchi-u.ac.jp

'Department of Digestive Surgery and Surgical Oncology, Yamaguchi University Graduate School of Medicine, Minami-Kogushi 1-1-1, Ube City, Yamaguchi Prefecture, Japan

Full list of author information is available at the end of the article
}

Campaign emphasized the importance of source control, adequate antibiotics therapy, and hemodynamic support in the early phase of sepsis management [6], which is termed early goal directed therapy [7]. Antibiotic and surgical therapies for source control are essential, but these approaches cannot remove endotoxins and the endogenous mediators already released into blood.

Polymyxin B-immobilized fiber (PMX) is a commercially available column that is covalently bound to polymyxin B (Toraymyxin; Toray Industries, Tokyo, Japan). Its structure prevents the elution of polymyxin B and protects patients from nephrotoxicity and neurotoxicity [8]. PMX is believed to mainly adsorb endotoxin, but 
recent studies have reported that it also adsorbs anandamide, inducing hypotension, immunosuppression, and cytotoxicity [9], and reduces serum cytokine levels $[10,11]$, monocyte messenger RNA expression [12], and the percentage of $\mathrm{CD} 4^{+} \mathrm{CD} 25^{+}$Forkhead box protein 3 (Foxp3) ${ }^{+} \mathrm{T}$ cells, termed regulatory $\mathrm{T}$ cells in the $\mathrm{CD} 4^{+}$ $T$ cell population [13].

It is known that direct hemoperfusion with PMX (PMX-DHP) has various effects on septic shock, and a few studies have shown that it has an ameliorating effect on prognosis [14]. Although a preliminary randomized controlled trial showed that PMX-DHP improved hemodynamic and respiratory dysfunction and reduced 28-day mortality in patients with severe sepsis and septic shock due to intra-abdominal infection [15], its improvement effect on prognosis remains controversial [16]. And two randomized controlled trials are ongoing in France (Effects of Hemoperfusion With a Polymyxin B Membrane in Peritonitis With Septic Shock (ABDOMIX); ClinicalTrials.gov identifier: NCT01222663) and the United States (Safety and Efficacy of Polymyxin B Hemoperfusion (PMX) for Septic Shock (EUPHRATES); ClinicalTrials.gov identifier: NCT01046669). Before discussing the role of PMX-DHP in the management of patients with severe sepsis and septic shock, we should consider the forthcoming results of these ongoing trials.

It is unclear which patients will benefit from PMXDHP and survive septic shock. If we could predict the prognosis of septic shock patients who undergo PMXDHP, we would be able to identify the patient in danger of death and make risk stratification for future novel therapies. To determine the predictive factors of survival in the early phase after starting PMX-DHP and make clinical prediction rule for survival, we conducted a retrospective analysis of patients with septic shock who underwent PMX.

\section{Methods}

\section{Patients}

Twenty-nine consecutive patients with septic shock who underwent PMX-DHP between January 2006 and December 2013 in the Department of Digestive Surgery and Surgical Oncology at Yamaguchi University Hospital were enrolled in this retrospective study. This study was approved by the institutional review board of Yamaguchi University. The review board approved the waiving of the informed consent because this study consisted of a historical cohort.

All patients were followed up until discharge or death. The patients were divided into groups based on survival $(n=23)$ and non-survival $(n=6) 28$ days after PMXDHP, and the clinical data for the two groups before and after PMX-DHP were compared.

\section{Initial resuscitation}

Administration of catecholamine was started to keep the patient's mean arterial pressure (MAP) $\geq 65 \mathrm{mmHg}$ despite adequate fluid resuscitation. We used noradrenaline as first-choice vasopressor and added vasopressin (0.03 $\mathrm{U} / \mathrm{min}$ ) if needed. Dobutamine infusion was added to vasopressor in patients with myocardial dysfunction [6]. Procedures for source control were conducted as soon as possible, if feasible. The presumptive therapies with broad spectrum antibiotics were started as soon as possible, and switched to definitive therapies with narrower spectrum agents after culture results and antimicrobial susceptibility data returned.

\section{PMX-DHP}

PMX-DHP was performed for patients with hemodynamic instability in spite of administration of catecholamine through a double-lumen catheter inserted in the cervical vein or the femoral vein at a blood flow rate of 80 $100 \mathrm{~mL} / \mathrm{min}$ with $20 \mathrm{mg} / \mathrm{h}$ nafamostat mesilate, as the anticoagulant. Treatment was performed for longer than $2 \mathrm{~h}$ and up to $24 \mathrm{~h}$ because some reports have suggested that an extended duration of treatment may be beneficial [17]. All patients underwent one or two sessions of PMXDHP.

\section{Clinical data}

Clinical data were recorded, and the Acute Physiology and Chronic Health Evaluation (APACHE II) score [18], Sequential Organ Failure Assessment (SOFA) score [19], and disseminated intravascular coagulation score from the Japanese Association of Acute Medicine [20] was determined at the start of PMX-DHP.

The inotropic score [21,22] is the most commonly used index in critical care medicine for expressing hemodynamic dysfunction and is calculated as follows (all doses are expressed as $\mu \mathrm{g} / \mathrm{kg} / \mathrm{min}$ ): (dopamine dose $\times 1)+$ (dobutamine dose $\times 1)+($ adrenaline dose $\times 100)+($ noradrenaline dose $\times 100)+($ phenylephrine dose $\times 100)$. Because this score has different meaning for targeted arterial pressure, we adopted the vasopressor dependency index (VDI) [15], which was introduced in the Early Use of Polymyxin B Hemoperfusion in Abdominal Sepsis (EUPHAS) trial and is calculated as the ratio of the inotropic score to the MAP.

The uses of continuous renal replacement therapy, antithrombin, recombinant-soluble thrombomodulin, and intravenous immunoglobulin were recorded. Procedures for source control included drainage for abdominal abscess.

We recorded the MAP, VDI, improvement rate of the VDI, leukocyte count, data on arterial blood gases, improvement rate of the $\mathrm{PaO}_{2} / \mathrm{FIO}_{2}$ ratio, and lactate clearance rate $6 \mathrm{~h}$ after the start of PMX-DHP. Although duration of PMX-DHP differed from patients to patients, 
we chose $6 \mathrm{~h}$ after the start of PMX-DHP instead of completion of that to evaluate every patient at the same point in time and determine the prognostic factors in the early phase after starting PMX-DHP.

\section{Statistical analysis}

Categorical variables are presented as numbers and were analyzed by using Fisher's exact test. Continuous variables were compared by using the Mann-Whitney $U$ test and data are presented as medians and ranges. We selected the predictive factors of 28-day survival, constructed receiver operating characteristic (ROC) curves of each factor and determined the area under the curve (AUC). The optimal cutoff point for balancing the sensitivity and specificity of each factor was identified as the point on the ROC curve closest to the upper left-hand corner. Subsequently, we allocated one point to each of the prognostic factor which was worse than the respective cutoff value, and calculated the total score of each patient, termed the prognostic score, by adding these points. The Kaplan-Meier method and log-rank test were performed for survival analysis of each score. We constructed ROC curve of the prognostic score for the prediction of 28-day survival and determined the AUC. A $p$ value $\leq 0.05$ was considered statistically significant. Data were analyzed with StatFlex version 6.0 (Artec, Osaka, Japan).

\section{Results}

\section{Baseline patient characteristics}

The demographic data before PMX-DHP are shown in Tables 1 and 2 . Of the 29 patients, 23 patients were alive and 6 patients had died 28 days after PMX-DHP. The proportion of cases of intra-abdominal infection was greater in survivors than in non-survivors $(p=0.013)$.

We did not conduct procedures for source control in five patients. There were two patients without identified

Table 1 Baseline patient characteristics

\begin{tabular}{|c|c|c|c|}
\hline & $\begin{array}{l}\text { Survivors } \\
(n=23)\end{array}$ & $\begin{array}{l}\text { Non-survivors } \\
(n=6)\end{array}$ & $P$ values \\
\hline Age (years) & $67.0(27-95)$ & $74.5(58-83)$ & 0.269 \\
\hline Gender (male/female) & $14 / 9$ & $4 / 2$ & 0.592 \\
\hline \multicolumn{4}{|l|}{$\begin{array}{l}\text { Site of infection (number } \\
\text { of patients) }\end{array}$} \\
\hline Intra-abdominal infection & 21 & 3 & 0.013 \\
\hline Pneumonia & 1 & 0 & \\
\hline unidentified source & 1 & 1 & \\
\hline CRBSI & 0 & 1 & \\
\hline Vibriovulnificus infection & 0 & 1 & \\
\hline $\begin{array}{l}\text { Procedures for source } \\
\text { control }(+/-)\end{array}$ & $20 / 3$ & $4 / 2$ & 0.269 \\
\hline
\end{tabular}

Data are presented as medians (ranges).

Abbreviations: CRBSI catheter-related bloodstream infection. source, a patient with pneumonia and a patient with systemic vibrio vulnificus infection. And a patient with abdominal abscess after hepatectomy did not undergo drainage because of marked bleeding tendency, and survived to discharge.

Of the 29 patients, 24 patients were treated with carbapenem or piperacillin/tazobactam agents as initial therapies. And vancomycin or daptomycin or linezolid were used concurrently if methicillin-resistant Staphylococcus aureus or Enterococcus species infection were possible pathogens.

There were no differences between the two groups in use of antithrombin, recombinant soluble thrombomodulin, and intravenous immunoglobulin (data not shown).

\section{Comparison of the clinical features and laboratory data before PMX-DHP between survivors and non-survivors} In non-survivors, the VDI before PMX-DHP was significantly higher $(p=0.046)$ and the leukocyte count before PMX-DHP was significantly lower $(p=0.024)$ than in survivors (Tables 3 and 4). We were not able to evaluate which population of leukocytes decreased in nonsurvivors because differential leukocyte counts were not consistently available. There were no other significant differences between the two groups.

\section{Comparison of clinical features $6 \mathrm{~h}$ after the start of PMX-DHP between survivors and non-survivors}

The $\mathrm{pH}$ and base excess (BE) of arterial blood of nonsurvivors were significantly lower than those of survivors ( $p=0.018$ and 0.007 , respectively) (Table 5). The lactate level was significantly higher in non-survivors $(p=0.043)$. The VDI after PMX-DHP decreased significantly $(p=0.0036)$ compared with the VDI before PMX-DHP only in survivors (data not shown). There was no significant difference in leukocyte count between survivors and non-survivors. And it did not show significant changes between before- and after PMX-DHP in both groups (data not shown). There were no significant differences in the VDI, improvement rate of the VDI, improvement rate of the $\mathrm{PaO}_{2}$ / $\mathrm{FIO}_{2}$ ratio, and lactate clearance rate.

\section{Analysis of ROC curves}

We constructed ROC curves of the VDI (Figure 1a) and leukocyte count (Figure 1b) before PMX-DHP for the prediction of 28-day survival and determined the AUC. The AUC (95\% CI) of the ROC curves of the VDI and leukocyte count were $0.768(0.514-1.000)$ and 0.804 (0.551-1.000), respectively. The optimal cutoff points (sensitivity, specificity) of the VDI and leukocyte count were $0.499 / \mathrm{mmHg}(78.3 \%, 83.3 \%)$ and $1360 / \mu \mathrm{L}(100 \%$, $66.7 \%)$, respectively.

The BE, pH, and lactate level after PMX-DHP were significantly correlated, and regression coefficients between 
Table 2 Baseline patient characteristics

\begin{tabular}{llll}
\hline & $\begin{array}{l}\text { Survivors } \\
(\boldsymbol{n = 2 3 )}\end{array}$ & $\begin{array}{l}\text { Non-survivors } \\
(\boldsymbol{n}=\mathbf{6})\end{array}$ & P values \\
\hline ICU admission to PMX-DHP start (min) & $62(15-2,317)$ & $75(20-1,170)$ & 0.483 \\
Number of PMX-DHP per patient (1/2) & $19 / 4$ & $4 / 2$ & 0.363 \\
Time per treatment (min) & & & \\
$\quad 1^{\text {st }}$ session & $325(50-1,835)$ & $820(200-1,340)$ & 0.161 \\
$2^{\text {nd }}$ session & $1,080(420-1,440)$ & $1,372(1,330-1,414)$ & NA \\
Total time of PMX-DHP (min) & $360(50-2,880)$ & $1,330(490-2,455)$ & 0.091 \\
CRRT (+/-) & $14 / 9$ & $5 / 1$ & 0.302 \\
\hline
\end{tabular}

Data are presented as medians (ranges).

Abbreviations: ICU intensive care unit, $P M X-D H P$ direct hemoperfusion with polymyxin B-immobilized fiber, NA not available, $C R R T$ continuous renal replacement therapy.

$\mathrm{BE}$ and $\mathrm{pH}, \mathrm{pH}$ and lactate level, and $\mathrm{BE}$ and lactate level were $0.8095,-0.5167$, and -0.6373 , respectively. To compare the prediction abilities of these factors, we constructed ROC curves. Because the AUC of the ROC curve of $\mathrm{BE}$ was greater than that of $\mathrm{pH}$ and lactate level $(\mathrm{BE}$ : 0.866 (95\% CI 0.647-1.000), pH: 0.819 (95\% CI 0.6380.999), lactate level: 0.772 (95\% CI $0.505-1.000)$ ), we considered $\mathrm{BE}$ to be the superior prognostic factor. We judged the optimal cutoff point of BE after PMX-DHP to be $-6.4 \mathrm{mmol} / \mathrm{L}$, and the sensitivity and specificity at this point were $95.7 \%$ and $83.3 \%$, respectively (Figure 1c).

\section{Prognostic score and 28-day survival}

Subsequently, we allocated one point to each of the three prognostic factors (VDI before PMX-DHP greater than $0.499 / \mathrm{mmHg}$, leukocyte count before PMX-DHP less than $1360 / \mu \mathrm{L}$, and BE after PMX-DHP less than $-6.4 \mathrm{mmol} / \mathrm{L})$, and calculated the prognostic score by adding these points. The 28-day survival rate for each score is shown in Figure 2. All patients with a score of $0(n=18)$ were alive after 28 days. The survival rates of patients with a score of $1(n=6)$ and a score of $2(n=4)$ were $67 \%$ and $25 \%$, respectively. The patient with a score of $3(n=1)$ died within $24 \mathrm{~h}$ after the start of PMX-DHP. There were significant differences in survival rates between a score of 0 and a score of $1(p=0.0078)$ and between a score of 0 and a score of $2(p<0.0001)$. The number of patients with a score of 3 was too small for statistical analysis.

The ROC curve of the prognostic score for the prediction of 28-day survival is shown in Figure 3. The AUC (95\% CI) was 0.946 (95\% CI $0.865-1.000)$ and greater than those of each three factors.

\section{Discussion}

The findings of this retrospective study suggest that the VDI and leukocyte count before PMX-DHP and BE after PMX-DHP may be prognostic factors, and the total score calculated with cutoff points for these factors could be related to the survival rate of patients with septic shock who undergo PMX-DHP. Some studies have reported prognostic factors in these patients, including APACHE II score [23,24], SOFA score [23], and HMGB1 [25]. To the best of our knowledge, this is the first report suggesting that VDI and leukocyte count before PMX-DHP and BE after PMX-DHP are prognostic

Table 3 Comparison of the clinical features before PMX-DHP between survivors and non-survivors

\begin{tabular}{llll}
\hline & $\begin{array}{l}\text { Survivors } \\
(\boldsymbol{n}=\mathbf{2 3})\end{array}$ & $\begin{array}{l}\text { Non-survivors } \\
(\boldsymbol{n}=\mathbf{6})\end{array}$ & $\begin{array}{c}\boldsymbol{P} \text { values } \\
\text { Respiratory rate (/min) }\end{array}$ \\
Bladder temperature (Celsius) & $16(10-25)$ & $16.5(12-30)$ & 0.745 \\
Heart rate (/min) & $36.9(34.2-38.8)$ & $36.2(34.4-37.9)$ & 0.536 \\
MAP (mmHg) & $105(67-135)$ & $120(80-130)$ & 0.186 \\
Inotropic score & $70(50-117)$ & $59(40-82)$ & 0.100 \\
VDI (/mmHg) & $14.1(2.3-44.7)$ & $45.5(5.5-50.6)$ & 0.095 \\
APACHE II score & $0.195(0.025-0.645)$ & $0.562(0.080-0.802)$ & 0.046 \\
SOFA score & $15(8-26)$ & $22(8-29)$ & 0.177 \\
DIC score & $7(2-13)$ & $8.5(6-15)$ & 0.099 \\
\hline
\end{tabular}

Data are presented as medians (ranges).

Abbreviations: MAP mean arterial pressure, VDI vasopressor dependency index, APACHE II acute physiology and chronic health evaluation, SOFA sequential organ failure assessment, DIC disseminated intravascular coagulation. 
Table 4 Comparison of the laboratory data before PMX-DHP between survivors and non-survivors

\begin{tabular}{|c|c|c|c|}
\hline & $\begin{array}{l}\text { Survivors } \\
(n=23)\end{array}$ & $\begin{array}{l}\text { Non-survivors } \\
(n=6)\end{array}$ & $P$ values \\
\hline 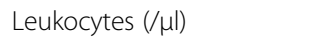 & $6,420(1,370-25,220)$ & $1,110(430-16,540)$ & 0.024 \\
\hline $\mathrm{Ht}(\%)$ & $33.3(23.8-44.9)$ & $34.4(19.2-45.5)$ & 0.957 \\
\hline Platelets $\left(\times 10^{4} / \mu \mathrm{l}\right)$ & $14.3(3.2-42.0)$ & $8.5(5.2-19.1)$ & 0.053 \\
\hline Total bilirubin (mg/dl) & $0.6(0.4-8.2)$ & $1.0(0.8-2.0)$ & 0.110 \\
\hline Creatinine (mg/dl) & $0.98(0.36-3.36)$ & $1.18(0.72-2.82)$ & 0.132 \\
\hline D-dimer ( $\mu \mathrm{g} / \mathrm{ml})$ & $11.2(1.0-502.9)$ & $7.8(3.7-16.6)$ & 0.448 \\
\hline C-reactive protein $(\mathrm{mg} / \mathrm{dl})$ & $6.75(0.26-27.38)$ & $7.07(0.34-13.05)$ & 0.477 \\
\hline \multicolumn{4}{|l|}{ Arterial blood gases } \\
\hline $\mathrm{pH}$ & 7.354 (7.249-7.469) & 7.325 (7.208-7.404) & 0.258 \\
\hline Base excess (mmol/l) & $-3.80(-10.2-3.1)$ & $-6.10(-8.10-1.60)$ & 0.170 \\
\hline $\mathrm{P} / \mathrm{F}(\mathrm{mmHg})$ & $229(91-525)$ & $195(102-395)$ & 0.360 \\
\hline Lactate $(\mathrm{mmol} / \mathrm{l})$ & $3.3(0.6-8.7)$ & $4.8(1.7-7.8)$ & 0.389 \\
\hline
\end{tabular}

Data are presented as medians (ranges).

Abbreviation: $\mathrm{P} / \mathrm{F} \mathrm{PaO}_{2} / \mathrm{FIO}_{2}$ ratio.

markers and the predictive score combined with these three factors.

The VDI, which was introduced in the EUPHAS trial [15], is a surrogate marker of hemodynamic status expressing a relationship between the dose of catecholamine and the response of MAP. There were no differences in MAP and inotropic score in this study, whereas the VDI in nonsurvivors was significantly higher than that in survivors. This result suggests that the VDI is a more sensitive marker of hemodynamic in patients with septic shock than the inotropic score. Kobayashi et al. reported that early improvement of VDI after PMX-DHP was a prognostic factor and a significant decrease in the VDI after PMX-DHP was seen only in survivors [26]. Because it is impossible to judge whether decreasing of the VDI in each patient is significant, we need to set a cutoff point or evaluate the improvement rate of the VDI for prediction of prognosis. The AUC of the ROC curve of the VDI after PMX-DHP in our study for the prediction of 28-day survival was 0.739 (95\% CI 0.544-0.934) and inferior to that before PMXDHP. However, the cutoff point of the VDI before PMXDHP determined in our study was also not sufficient for prognostic prediction; these results indicate that the VDI may be just one aspect of the severity of sepsis.

There were no significant differences in VDI and improvement rate of the VDI between the two groups $6 \mathrm{~h}$ after the start of PMX-DHP in this study. We think two main reasons for this. First, sample size was too small to detect significance because VDI in non-survivors after PMX-DHP tended to be higher than that in survivors (Table 5). Second, microcirculatory dysfunction could not be improved only by hemodynamic stabilization.

Table 5 Comparison of clinical features $6 \mathrm{~h}$ after the start of PMX-DHP between survivors and non-survivors

\begin{tabular}{|c|c|c|c|}
\hline & $\begin{array}{l}\text { Survivors } \\
(n=23)\end{array}$ & $\begin{array}{l}\text { Non-survivors } \\
(n=6)\end{array}$ & $P$ values \\
\hline MAP (mmHg) & $75(45-101)$ & $79(42-98)$ & 0.914 \\
\hline VDI (/mmHg) & $0.106(0.000-0.569)$ & $0.256(0.130-0.612)$ & 0.076 \\
\hline Improvement rate of VDI (\%) & $39.3(-116.9-100.0)$ & $39.2(-61.4-74.1)$ & 0.957 \\
\hline Leukocytes $(/ \mu l)$ & $5,700(1,050-31,200)$ & $3,800(1,400-11,100)$ & 0.200 \\
\hline \multicolumn{4}{|l|}{ Arterial blood gas } \\
\hline $\mathrm{pH}$ & 7.395 (7.305-7.458) & $7.329(7.130-7.388)$ & 0.018 \\
\hline Base excess (mmol/l) & $-1.7(-6.7-5.5)$ & $-7.2(-12.9-0.2)$ & 0.007 \\
\hline $\mathrm{P} / \mathrm{F}(\mathrm{mmHg})$ & $267(108-483)$ & $222(50-379)$ & 0.132 \\
\hline Improvement rate of $\mathrm{P} / \mathrm{F}(\%)$ & $9.0(-44.8-228.2)$ & $59.2(-76.5-169.8)$ & 0.518 \\
\hline Lactate $(\mathrm{mmol} / \mathrm{l})$ & $2.9(0.8-8.8)$ & $6.5(1.3-9.9)$ & 0.043 \\
\hline Lactate clearance (\%) & $10.0(-83.3-61.9)$ & $-6.6(-65.0-23.5)$ & 0.060 \\
\hline
\end{tabular}

Data are presented as medians (ranges).

Abbreviations: MAP mean arterial pressure, $\mathrm{VDI}$ vasopressor dependency index, $\mathrm{P} / \mathrm{F} \mathrm{PaO}_{2} / \mathrm{F} 1 \mathrm{O}_{2}$ ratio. 


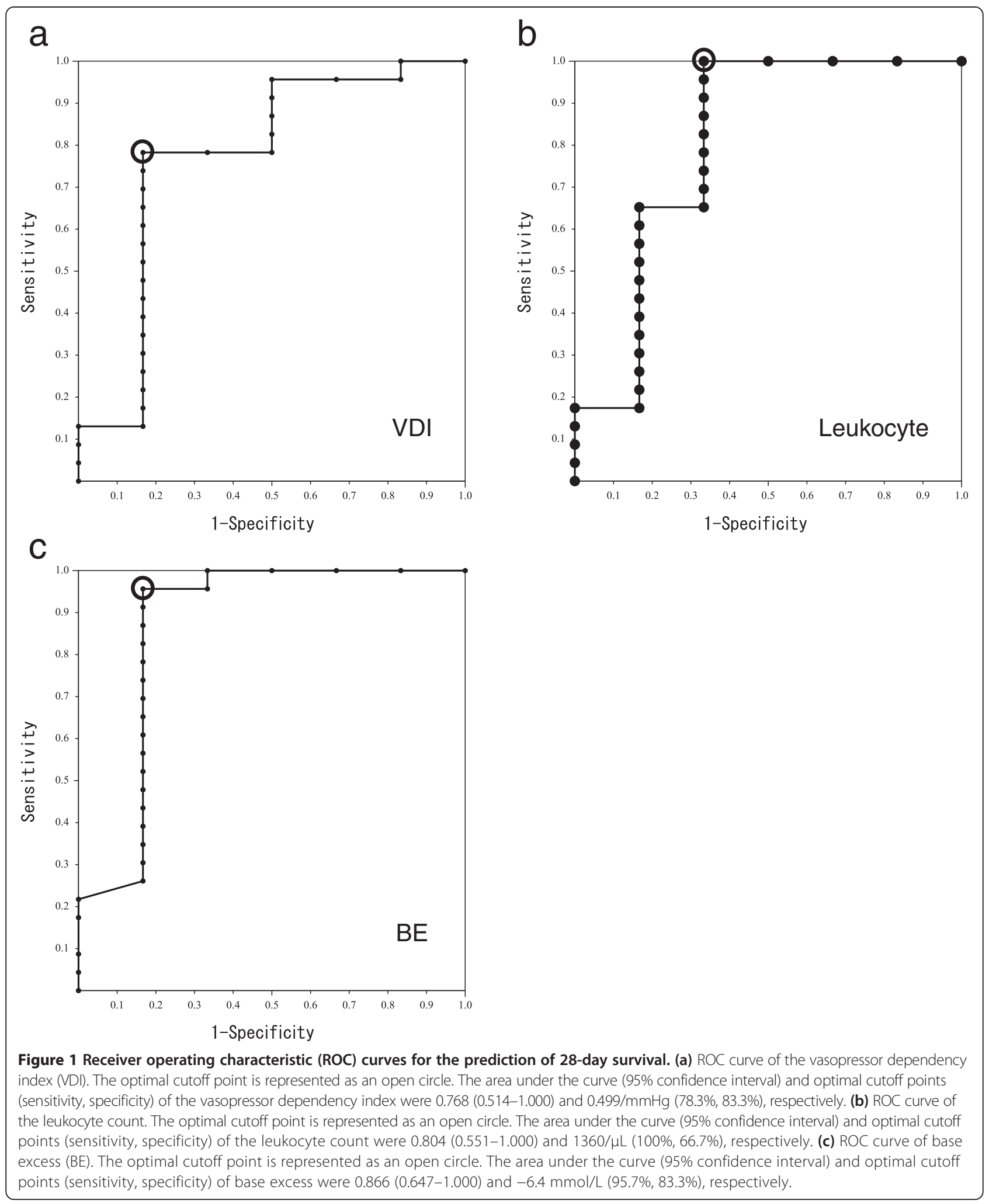

A decreased peripheral blood leukocyte count in the early phase of sepsis has been explained as the migration of leukocytes to the focus of infection or some organs induced by several cytokines, and it is an important feature for the diagnosis of sepsis [27]. Leukopenia has been reported as a prognostic factor of sepsis in some 


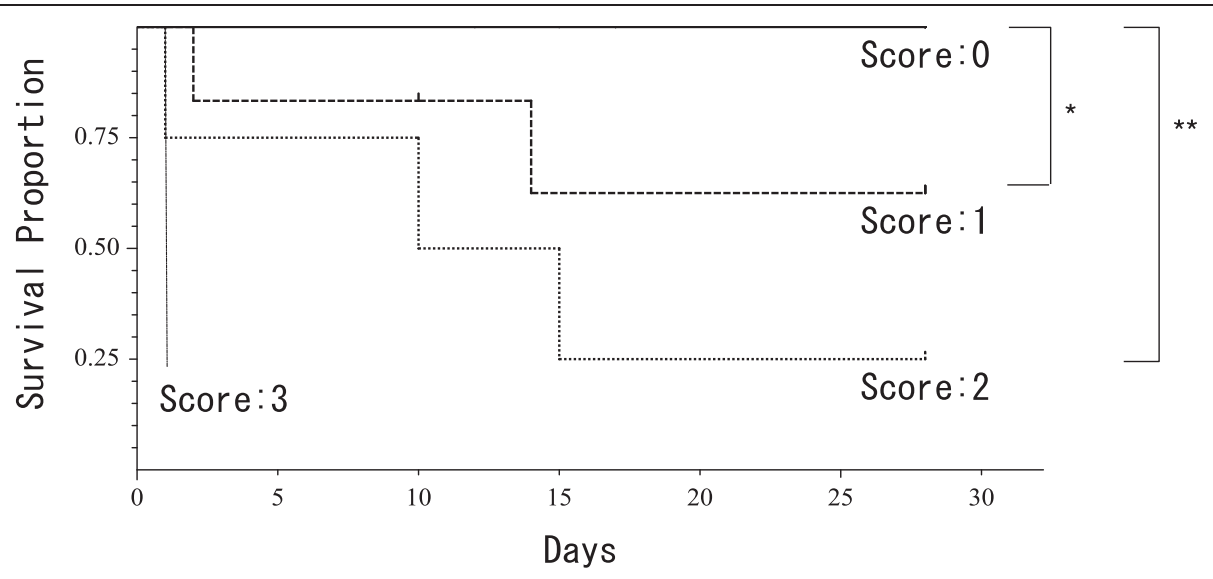

$$
{ }^{*}: p<0.01,{ }^{* *}: p<0.001
$$

Figure 2 Survival rate of each group. We allocated one point to each of the three prognostic factors (vasopressor dependency index before PMX-DHP greater than $0.499 / \mathrm{mmHg}$, leukocyte count before PMX-DHP less than 1360/ $\mathrm{LL}$, and base excess after PMX-DHP less than -6.4 mmol/L), and calculated the prognostic score by adding these points. All patients with a score of $0(n=18)$ were alive after 28 days, whereas the patient with a score of $3(n=1)$ died within $24 \mathrm{~h}$. The survival rates of the patients with a score of $1(n=6)$ and a score of $2(n=4)$ were $67 \%$ and $25 \%$, respectively. There were significant differences in survival rates between a score of 0 and a score of $1(p=0.0078)$ and between a score of 0 and a score of $2(p<0.0001)$. Abbreviation: PMX-DHP, direct hemoperfusion with polymyxin B-immobilized fiber.

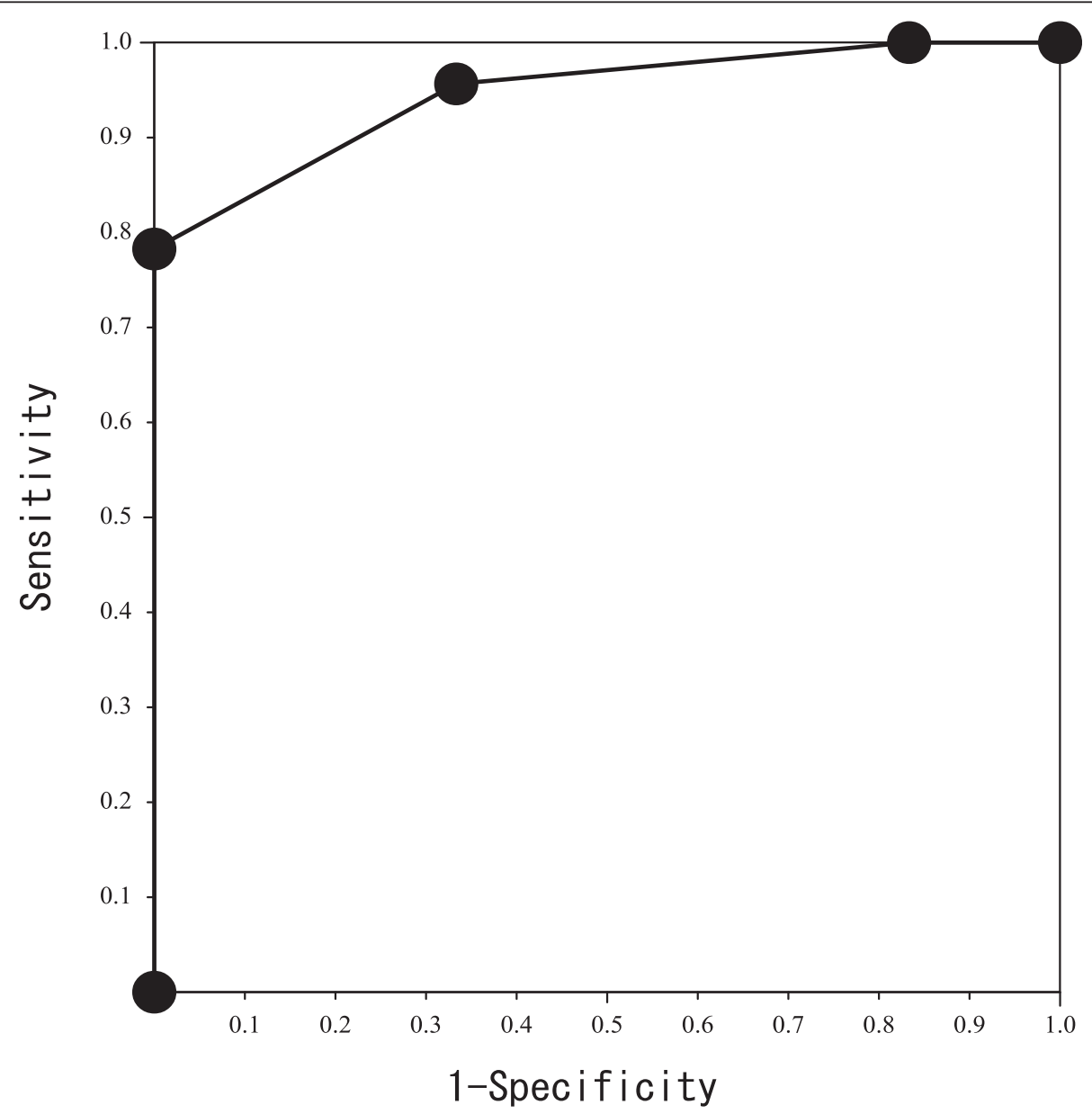

Figure 3 Receiver operating characteristic curve of the prognostic score for the prediction of 28-day survival. The area under the curve (95\% confidence interval) was $0.946(0.865-1.000)$ and greater than those of each three factors. 
studies [28], and our finding suggests that it may also be important in patients with septic shock who have undergone PMX-DHP. Unfortunately, the differential leukocyte count was not assessed in almost half of our patients, so it is unknown which population is especially important for prognosis. Because leukopenia in patients with sepsis means that hematopoietic function cannot respond to inflammation, immunodeficiency could develop in these patients.

Acidemia, decreased BE, and hyperlactemia are consequences of tissue hypoperfusion and imbalance of oxygen delivery and consumption. Of these three factors, lactate has been focused on in the past. Lactate-guided therapy significantly reduces hospital mortality [29], and the Surviving Sepsis Campaign guideline recommends normalizing the lactate level as rapidly as possible [6]. On the other hand, Couto-Alves et al. have reported a scoring system using $\mathrm{BE}$ and platelet count at presentation for prognostic prediction in pediatric meningococcal sepsis [30]. Although the lactate level was also significantly higher among non-survivors in that study, BE was used to build a score because of its better sensitivity. The reason why $\mathrm{BE}$ was more sensitive than lactate is unknown, but this discrepancy could reflect increased fixed acids by impaired excretion that resulted from renal dysfunction or hypercatabolism caused by inflammation.

In this study, the proportion of septic patients with intra-abdominal infection was significantly higher in the survivors. Although there was no difference between survivors and non-survivors regarding whether procedure was performed, the fact that surgical procedures were more available for intra-abdominal infection compared with other infectious sources may improve survival. In addition, PMX-DHP may be more useful for intra-abdominal sepsis, as reflected in many studies of PMX-DHP designed for intra-abdominal sepsis alone.

We acknowledge that this study has some limitations. First, a small number of patients with septic shock attributable to various causes were analyzed, and we could not perform multivariate analysis because of the limited number of patients. For this reason, these findings cannot be generalized to the broader clinical situation based on this study alone. Second, the effectiveness of PMX-DHP itself is uncertain because this study was retrospective. Third, the predictive score, which was calculated by adding one point for each factor, is insufficient because the weighting of each factor was not considered. We need to identify prognostic factors in well-designed, prospective, randomized, controlled trials with a large number of patients.

\section{Conclusions}

In conclusion, the VDI and leukocyte count at the start of PMX-DHP and BE $6 \mathrm{~h}$ after the start of PMX-DHP are related to prognosis of patients with septic shock who undergo PMX-DHP. The prognostic score using the respective cutoff values of these three factors may be useful to predict the prognosis of these patients.

\section{Competing interests}

The authors declare that they have no competing interests.

\section{Authors' contributions}

SM conceived the study, carried out the study, and drafted the manuscript. KS participated in the study's design and interpretation of data, and helped to draft the manuscript. MN, T, RO, NM, SM, and HW collected the data and helped to interpret the data and draft the manuscript. SY, SH, RT, MM, and $\mathrm{MO}$ revised the draft and supervised and edited the study. All authors read and approved the final manuscript.

\section{Acknowledgements}

The authors thank Mr. Kenichi Hiraga, a clinical engineer, for technical assistance and data management as well as all the nurses, physicians, and other personnel in the intensive care unit for their dedicated cooperation.

\section{Author details}

'Department of Digestive Surgery and Surgical Oncology, Yamaguchi University Graduate School of Medicine, Minami-Kogushi 1-1-1, Ube City, Yamaguchi Prefecture, Japan. Intensive Care Unit, Yamaguchi University Hospital, Ube City, Yamaguchi Prefecture, Japan. ${ }^{3}$ Advanced Medical Emergency and Critical Care Center, Yamaguchi University Hospital, Ube City, Yamaguchi Prefecture, Japan.

Received: 6 November 2014 Accepted: 19 February 2015

Published online: 13 March 2015

\section{References}

1. Levy MM, Fink MP, Marshall JC, Abraham E, Angus D, Cook D, et al. 2001 SCCM/ESICM/ACCP/ATS/SIS International Sepsis Definitions Conference. Crit Care Med. 2003;31:1250-6.

2. Adhikari NK, Fowler RA, Bhagwanjee S, Rubenfeld GD. Critical care and the global burden of critical illness in adults. Lancet. 2010;376:1339-46.

3. Angus DC, Linde-Zwirble WT, Lidicker J, Clermont G, Carcillo J, Pinsky MR. Epidemiology of severe sepsis in the United States: analysis of incidence, outcome, and associated costs of care. Crit Care Med. 2001;29:1303-10.

4. Kumar G, Kumar N, Taneja A, Kaleekal T, Tarima S, McGinley E, et al. Nationwide trends of severe sepsis in the 21st century (2000-2007). Chest. 2011;140:1223-31.

5. Lagu T, Rothberg MB, Shieh MS, Pekow PS, Steingrub JS, Lindenauer PK. Hospitalizations, costs, and outcomes of severe sepsis in the United States 2003 to 2007. Crit Care Med. 2012;40:754-61.

6. Dellinger RP, Levy MM, Rhodes A, Annane D, Gerlach H, Opal SM, et al. Surviving Sepsis Campaign: international guidelines for management of severe sepsis and septic shock, 2012. Intensive Care Med. 2013;39:165-228.

7. Rivers E, Nguyen B, Havstad S, Ressler J, Muzzin A, Knoblich B, et al. Early goal-directed therapy in the treatment of severe sepsis and septic shock. N Engl J Med. 2001;345:1368-77.

8. Ronco C. Endotoxin removal: history of a mission. Blood Purif. 2014;37 Suppl 1:5-8.

9. Wang Y, Liu Y, Sarker KP, Nakashima M, Serizawa T, Kishida A, et al. Polymyxin $B$ binds to anandamide and inhibits its cytotoxic effect. FEBS Lett. 2000;470:151-5.

10. Tani T, Hanasawa K, Kodama M, Imaizumi H, Yonekawa M, Saito M, et al. Correlation between plasma endotoxin, plasma cytokines, and plasminogen activator inhibitor-1 activities in septic patients. World J Surg. 2001;25:660-8.

11. Nakamura T, Ebihara I, Shimada N, Koide H. Changes in plasma erythropoietin and interleukin-6 concentrations in patients with septic shock after hemoperfusion with polymyxin B-immobilized fiber. Intensive Care Med. 1998;24:1272-6.

12. Nakamura $\mathrm{T}$, Ebihara I, Shimada $\mathrm{N}$, Shoji $\mathrm{H}$, Koide $\mathrm{H}$. Modulation of plasma metalloproteinase-9 concentrations and peripheral blood monocyte mRNA levels in patients with septic shock: effect of fiber-immobilized polymyxin B treatment. Am J Med Sci. 1998;316:355-60. 
13. Ono S, Kimura A, Hiraki S, Takahata R, Tsujimoto H, Kinoshita M, et al. Removal of increased circulating CD4 + CD25 + Foxp3+ regulatory T cells in patients with septic shock using hemoperfusion with polymyxin B-immobilized fibers. Surgery. 2013;153:262-71.

14. Zhou F, Peng Z, Murugan R, Kellum JA. Blood purification and mortality in sepsis: a meta-analysis of randomized trials. Crit Care Med. 2013;41:2209-20.

15. Cruz DN, Antonelli M, Fumagalli R, Foltran F, Brienza N, Donati A, et al. Early use of polymyxin B hemoperfusion in abdominal septic shock: the EUPHAS randomized controlled trial. JAMA. 2009;301:2445-52.

16. Iwagami M, Yasunaga H, Doi K, Horiguchi H, Fushimi K, Matsubara T, et al. Postoperative polymyxin $B$ hemoperfusion and mortality in patients with abdominal septic shock: a propensity-matched analysis. Crit Care Med. 2014:42:1187-93.

17. Mitaka C, Tsuchida N, Kawada K, Nakajima Y, Imai T, Sasaki S. A longer duration of polymyxin B-immobilized fiber column hemoperfusion improves pulmonary oxygenation in patients with septic shock. Shock. 2009;32:478-83.

18. Knaus WA, Draper EA, Wagner DP, Zimmerman JE. APACHE II: a severity of disease classification system. Crit Care Med. 1985;13:818-29.

19. Vincent JL, Moreno R, Takala J, Willatts S, De Mendonca A, Bruining H, et al. The SOFA (Sepsis-related Organ Failure Assessment) score to describe organ dysfunction/failure. On behalf of the Working Group on Sepsis-Related Problems of the European Society of Intensive Care Medicine. Intensive Care Med. 1996:22:707-10.

20. Gando S, Iba T, Eguchi Y, Ohtomo Y, Okamoto K, Koseki K, et al. A multicenter, prospective validation of disseminated intravascular coagulation diagnostic criteria for critically ill patients: comparing current criteria. Crit Care Med. 2006;34:625-31.

21. Shore S, Nelson DP, Pearl JM, Manning PB, Wong H, Shanley TP, et al. Usefulness of corticosteroid therapy in decreasing epinephrine requirements in critically ill infants with congenital heart disease. Am J Cardiol. 2001;88:591-4.

22. Wernovsky G, Wypij D, Jonas RA, Mayer Jr JE, Hanley FL, Hickey PR, et al. Postoperative course and hemodynamic profile after the arterial switch operation in neonates and infants. A comparison of low-flow cardiopulmonary bypass and circulatory arrest. Circulation. 1995;92:2226-35.

23. Komatsu S, Shimomatsuya T, Nakajima M, Ono S, Maruhashi K. Severity scoring systems for prognosis and efficacy of polymyxin B-immobilized fiber treatment for colonic perforation. Surg Today. 2006;36:807-10.

24. Sugimoto K, Sato K, Maekawa H, Sakurada M, Orita H, Ito T, et al. Analysis of the efficacy of direct hemoperfusion with polymyxin B-immobilized fiber (PMX-DHP) according to the prognostic factors in patients with colorectal perforation. Surg Today. 2013:43:1031-8.

25. Ueno T, Ikeda T, Ikeda K, Taniuchi H, Suda S, Yeung MY, et al. HMGB-1 as a useful prognostic biomarker in sepsis-induced organ failure in patients undergoing PMX-DHP. J Surg Res. 2011;171:183-90.

26. Kobayashi A, Iwasaki Y, Kimura Y, Kawagoe $Y$, Ujike $Y$. Early recovery in hemodynamics after direct hemoperfusion with polymyxin B-immobilized fibers may predict mortality rate in patients with septic shock. J Anesth. 2010;24:709-15.

27. Bone RC, Balk RA, Cerra FB, Dellinger RP, Fein AM, Knaus WA, et al. Definitions for sepsis and organ failure and guidelines for the use of innovative therapies in sepsis. The ACCP/SCCM Consensus Conference Committee. Am Coll Chest Physicians/Soc Crit Care Med Chest. 1992;101:1644-55

28. Wester AL, Dunlop O, Melby KK, Dahle UR, Wyller TB. Age-related differences in symptoms, diagnosis and prognosis of bacteremia. BMC Infect Dis. 2013;13:346

29. Jansen TC, van Bommel J, Schoonderbeek FJ, Sleeswijk Visser SJ, van der Klooster JM, Lima AP, et al. Early lactate-guided therapy in intensive care unit patients: a multicenter, open-label, randomized controlled trial. Am J Respir Crit Care Med. 2010;182:752-61.

30. Couto-Alves A, Wright VJ, Perumal K, Binder A, Carrol ED, Emonts M, et al. A new scoring system derived from base excess and platelet count at presentation predicts mortality in paediatric meningococcal sepsis. Crit Care. 2013;17:R68.

\section{Submit your next manuscript to BioMed Central and take full advantage of:}

- Convenient online submission

- Thorough peer review

- No space constraints or color figure charges

- Immediate publication on acceptance

- Inclusion in PubMed, CAS, Scopus and Google Scholar

- Research which is freely available for redistribution

Submit your manuscript at www.biomedcentral.com/submit 